\title{
AIAA-2002-0936 Floating Potential Probe Langmuir Probe Data Reduction Results
}

\author{
T. L. Morton, OAI, Brook Park, OH \\ J. I. Minow, Sverdrup Technology, Huntsville, AL
}

This is a preprint or reprint of a paper intended for presentation at a conference. Because changes may be made before formal publication, this is made available with the understanding that it will not be cited or reproduced without the permission of the author.

\section{0th AIAA Aerospace Sciences Meeting \& Exhibit 14-17 January 2002 / Reno, NV}


AIAA-2002-0936

\section{FLOATING POTENTIAL PROBE LANGMUIR PROBE DATA REDUCTION RESULTS}

Thomas L. Morton*

OAI, 21000 Brookpark Road, Cleveland, MS 302-1, OH 44135

Joseph I. Minow**

Sverdrup Technology, Inc., Marshall Space Flight Center Group, MSFC/ED44, Huntsville, AL 35812

\begin{abstract}
$\underline{\text { Abstract }}$
During its first five months of operations, the Langmuir Probe on the Floating Potential Probe (FPP) obtained data on ionospheric electron densities and temperatures in the ISS orbit. In this paper, the algorithms for data reduction are presented, and comparisons are made of FPP data with ground-based ionosonde and Incoherent Scattering Radar (ISR) results. Implications for ISS operations are detailed, and the need for a permanent FPP on ISS is examined.
\end{abstract}

\section{Introduction}

In the early 90's, a series of studies [Ferguson et al., 1990] revealed that the Space Station (ISS) electrical potential would lie at dangerously low voltages, due to the high voltage solar arrays, and negative grounding scheme. That is, the ISS would change its electrical floating potential to maintain a net zero current to the ambient plasma. Since electrons are much lighter than oxygen ions (the predominant ion component of the low Earth orbit plasma), the electrical ground of the ISS would float so that the negative end of the solar arrays was about 120 volts below the plasma ground, and the positive end of the solar arrays was about 40 volts above the plasma ground.

Anodized surfaces of ISS modules, a non-conductive coating, will not affect the collection of electrons or ions from the ambient plasma. Since the modules are grounded to the negative end of the solar arrays, they will also lie 120 volts below the plasma ground. The impact of this is that the anodized aluminum surfaces could experience arcing and sputtering, with the possible loss of their thermal control properties [Carruth et al., 2001]. In order to alleviate this hazard, NASA decided to install a Plasma Contacting Unit (PCU) on the Space Station. This device emits a xenon plasma, and provides a conductive pathway from the Space Station to the ambient plasma [Chaky and Lambert, 1996].

* Senior Research Associate, OAI.

** Senior Engineer, Sverdrup Technology (MSFC Group), Member AIAA.
Copyright (C) 2002 The American Institute of Aeronautics and Astronautics Inc. All rights reserved.

However, in pre-flight testing, it was discovered that the PCU could fail to clamp the voltage while the diagnostic telemetry appeared normal. This indicated that the Space Station needed an independent method to verify that the floating potential was within the design guidelines. In addition, since this was needed quickly, it had to be built and tested on a very tight schedule.

In June, 2000, NASA decided to add a Langmuir Probe to measure the floating potential. Since its purpose was to measure the ISS floating potential, it was named the Floating Potential Probe (FPP). In order to get the probe built and space qualified in time for mission $4 \mathrm{~A}$, it was necessary to use hardware that was already space qualified. The Langmuir Probe came from the Solar Array Module Interaction Experiment (SAMPIE) flight hardware, which had flown on STS-62 in 1994 [Perez de la Cruz et al., 1996]. Other parts of the FPP are documented in a previous paper [Ferguson et al., 2001]

Although the primary purpose of the FPP is to measure the Floating Potential, the SAMPIE hardware included a second Langmuir Probe, which can measure the ambient plasma density and temperature. This paper details some of the results from that second Langmuir Probe.

We will first review the analysis of the Langmuir Probe data, and show some sample results, compared to the IRI models for the same times. Generally, the densities from FPP are consistent with IRI, but the temperatures are high. To determine if this is caused by the average nature of the IRI model, or if something is wrong with the FPP analysis, we also compare to Incoherent Scatter Radar measurements for April 12, which provide an independent measurement of the plasma parameters. These also show that the FPP temperatures are generally high, but this may be due to a very active ionosphere on April 12. Since there were no other dates for which ISR data is available for the same times as FPP data, we try to draw some conclusions from these results. 


\section{Analysis of Langmuir Probe Data}

An extended discussion of the analysis of the Langmuir Probe is can be seen in a previous papers [Morton, et al., 1995; Ferguson et al., 2001]. The key issues that had to be resolved were 1) the Langmuir Probe measures only net negative current, and 2) the ion current is dominated by the ram velocity as the spacecraft moves through the plasma.

For the SAMPIE analysis, we used a model of Langmuir Probe current developed by Medicus [1961, 1962]. In essence, the total current collected by the probe is treated as the difference between the ram ion and thermal electron currents

$$
I_{\text {tot }}=I_{\text {ram, ion }}-I_{e}\left(n_{e}, T_{e}, V-V_{\text {plasmaa }}\right)
$$

where $n_{e}, T_{e}$, and $V_{\text {plasma }}$ represent the electron density, the electron temperature, and the plasma potential. The ram ion current can be written as

$$
I_{\text {ram, ion }}=n_{\text {ion }} \mathbf{v}_{s / c} q_{\text {ion }} A_{\text {probe }}
$$

where $n_{\text {ion }}$ is the ion density, $v_{s / c}$ is the spacecraft velocity, $\mathrm{q}_{\text {ion }}$ is the ionic charge, and $\mathrm{A}_{\text {probe }}$ is the crosssectional area of the Langmuir Probe. The Medicus papers provide the functional dependence of $I_{e}$ on $n_{e}$, $\mathrm{T}_{e}$, and $\mathrm{V}_{\text {plasma. }}$.

However, the probe cannot detect net positive currents, so there is no way to determine the ion density (or temperature). To get a reasonable solution, we assumed that the plasma is electrically neutral, so that $n_{\text {ion }}=n_{e}$. We are still left with a fitting procedure to extract the electron plasma parameters and the plasma potential from the Langmuir Probe trace, using Equation 1. In this case, the routine took an approximate density and temperature, and varied the plasma potential until the knee (the most curved part of the trace) of the fit lined up with the knee of the trace. Then, all three parameters were varied to fit the trace in the region of the knee. The reason the knee was chosen is that it determines the temperature, and temperature was the most difficult plasma parameter to obtain. A sample fit is shown in Figure 1.

However, when we applied the same technique to the FPP data, there was a lot of noise in the calculated densities and temperatures. When we looked at the data, we saw that there were discontinuities in the raw data, caused by an odd timing loop in the FPP firmware. In addition, sometimes the curve fitting

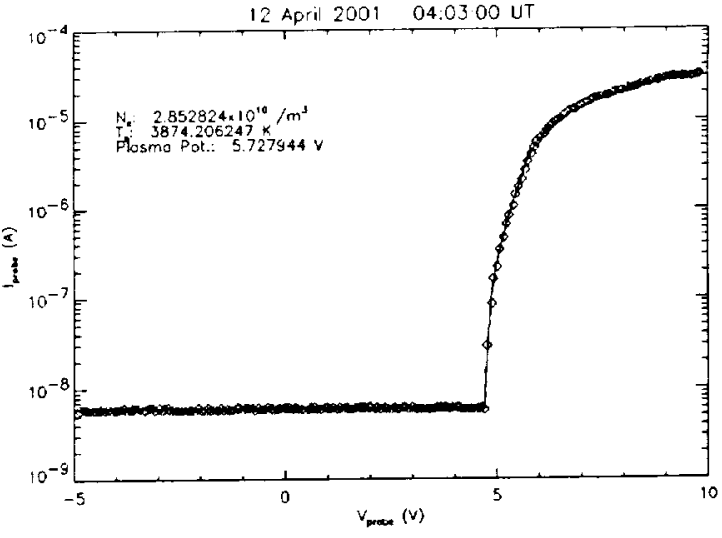

Figure 1. Langmuir Probe I-V curve. The fit (solid line) to the data (diamonds) in this example is excellent and results in the electron temperature and density values indicated in the plot.

algorithm would find a false minimum in it's minimization routine, and would not converge to the best density and temperature.

In order to not use these bad traces, we calculated a $\chi^{2}$ for each trace, and used only the traces with a small $\chi^{2}$. We also used the most the most recent good values of the density and temperature as the initial guess for succeeding data sets. This resulted in smoother curves, at the expense of some of the data points. Since we received a new trace every 20 seconds, this didn't seem to be a serious loss.

An additional limitation of the Langmuir Probe data was that it only scanned from -5 volts to +10 volts. If the Space Station was more then 10 volts lower than plasma ground, the Langmuir Probe would not receive any electron current. During some of the DTO's (Space Station experiments), the Space Station potential was more than 10 volts below the plasma ground. In those cases, we did not have a Langmuir Probe trace to evaluate. In addition, the last "good" value was not a good initial guess for the next available trace. We used the older method from the SAMPIE experiment to get an initial guess for the solution, and proceeded from there. In general, this worked fine.

\section{Comparison of FPP Records to IRI Model}

Figure 2 shows FPP measurements for approximately six orbits on 15 February 200 compared with the International Reference Ionosphere 1995 (IRI-95) model (Bilitza, 1994; 1997). This interval was selected 


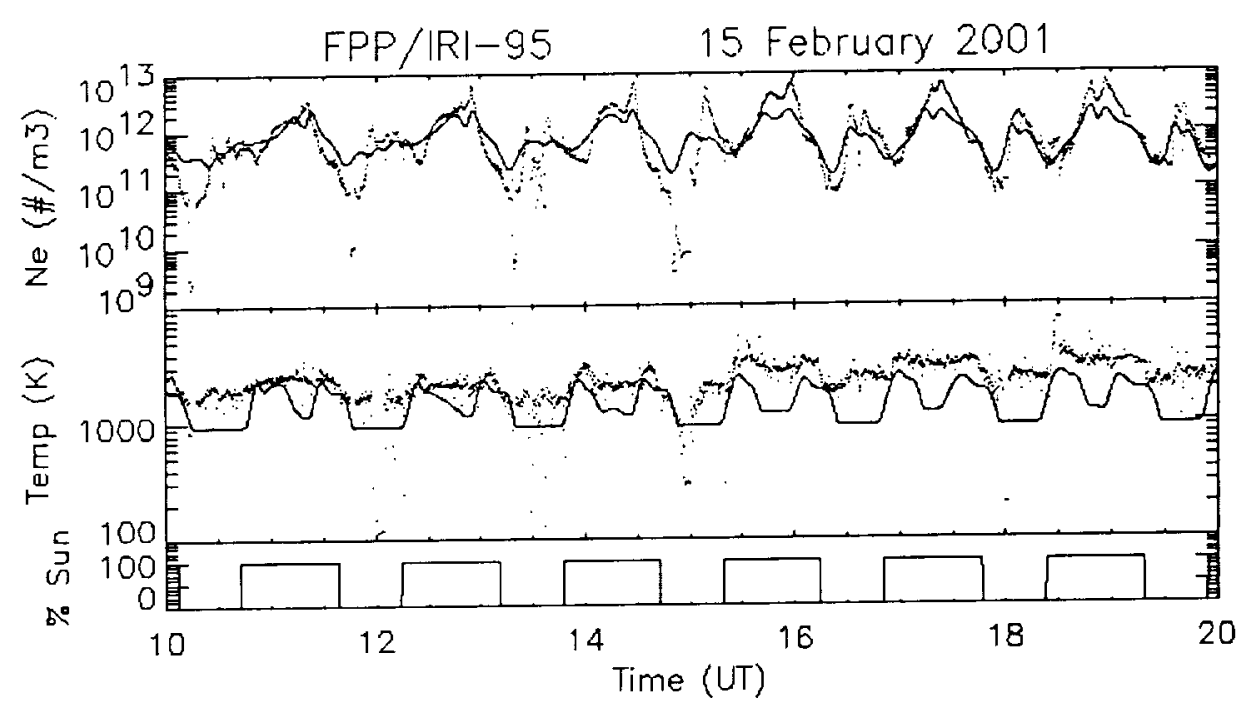

Figure 2. Comparison of FPP (dots) and IRI-95 model (solid) electron densities and temperatures. Values are plotted for a number of ISS orbits on 15 February 2001. Electron density values are shown in the top panel. The middle panel is the electron temperatures. Solar illumination is shown in the bottom panel where $100 \%$ illumination indicates orbital day and $0 \%$ orbital night.

as an example of available FPP data obtained during relatively undisturbed geomagnetic conditions. As shown in Figure 3, Kp values never exceeded 4 on 15 February with $\Sigma \mathrm{Kp}$ values of 270 and 107 on February 14 and 15 , respectively, consistent with quiet conditions during the observation period.

Plasma densities from the Langmuir probe compare favorably to the IRI values during orbital night periods and during the first few orbits from 10 UT through 15 UT. Isolated density peaks often exceed the IRI-95 predictions by a factor of 3 during orbital daytime. FPP

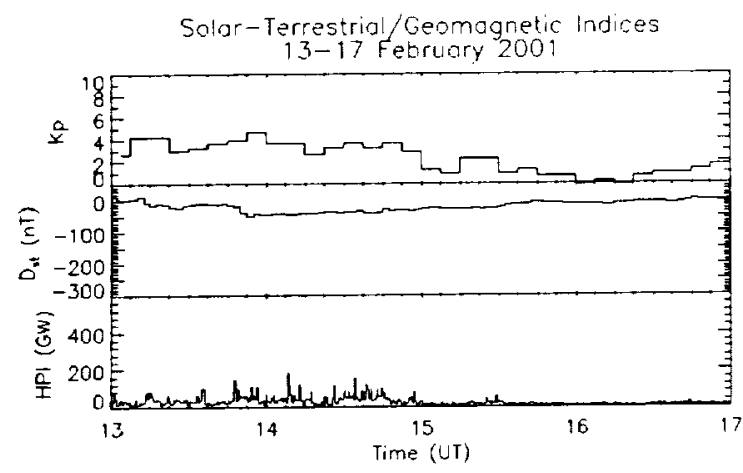

Figure 3. Geomagnetic conditions for 13-17 February 2001. Kp values are moderate to minor storm levels on 13 and 14 February but are quiet on February 15 when the FPP measurements in Figure 1 were obtained. observations of electron density values are generally greater than the IRI-95 model results throughout this interval.

Electron temperatures shown in the middle panel of Figure 2 consistently exceed the model values, often by $1000 \mathrm{~K}$ to $2000 \mathrm{~K}$, both during orbital day and night periods. Noise in the FPP temperature values is likely due to the combined effects of sensitivity in the fits to provide good temperature values and natural variations in electron temperature along the orbit.

Both the electron temperature and density values obtained by the FPP Langmuir Probe exhibit similar functional form to the IRI-95 model along the spacecraft orbit. Densities show greater deviations from the model values while the temperature measurements exhibit more noise and FPP measurements typically exceed the model results throughout the period.

Figure 4 compares FPP measurements of electron density and temperature and IRI-95 values for 12 April 2001. A DTO scheduled during this interval with the purpose of testing spacecraft charging models developed for the International Space Station assured that routine operation of the FPP would continue for several days with few or no interruptions. Fortuitously, a ground based research radar was monitoring the geomagnetic storm and provided an opportunity for coincident observations of plasma characteristics 


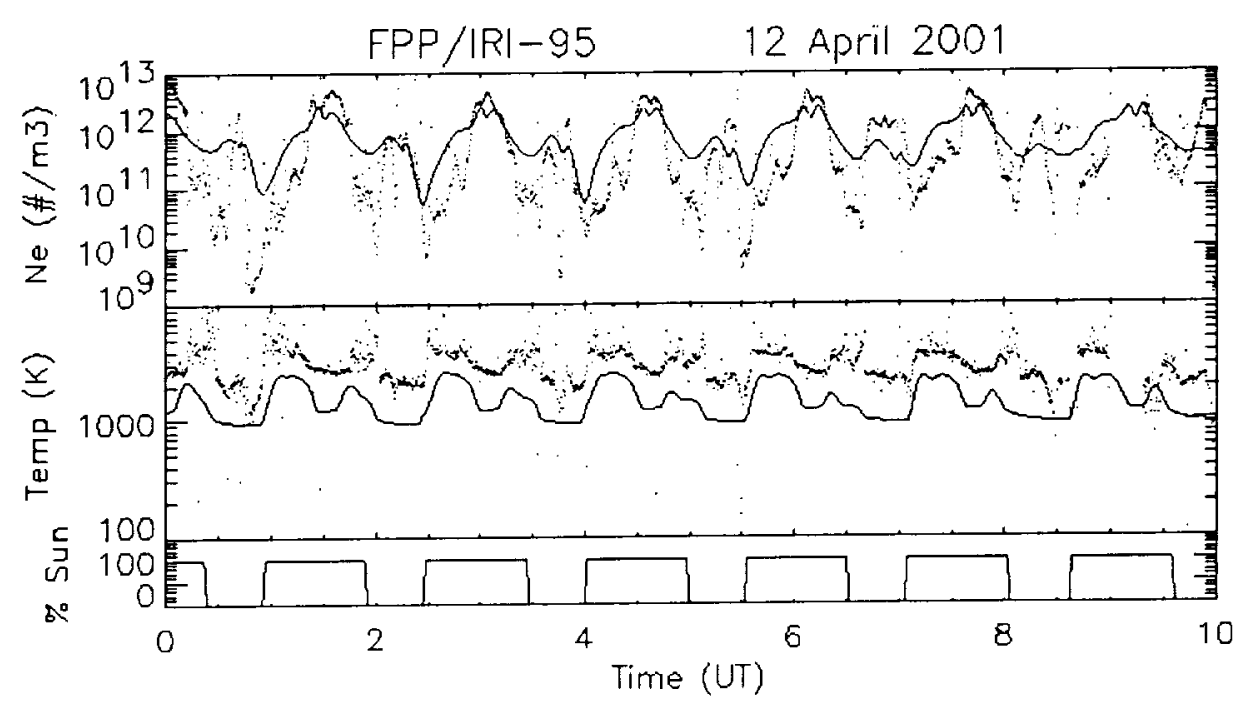

Figure 4. FPP (dots) and IRI-95 model (solid) electron densities and temperatures for 12 April 2001. The plot format is the same as I Figure 1. Model densities exceed many of the observed values while the model temperatures are less than the FPP observations during this geomagnetically disturbed period.

Geomagnetic conditions are disturbed during the DTO interval from 11-13 April 2001. Figure 5 shows $\mathrm{Kp}$ values exceeding $\mathrm{Kp}=6$ during much of the interval when the simultaneous radar and FPP observations were available. A large decrease in the Dst index indicates an increase in the ring current and the hemispheric power index demonstrates that significant particle precipitation was ongoing during the study interval, providing an energy source to disturb the high latitude plasma. Although it was this storm that was the focus of the ISR observations that provided the

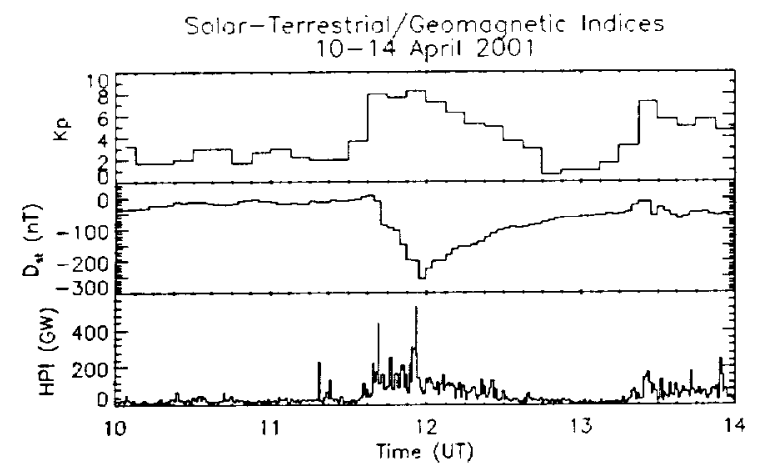

Figure 5. Geomagnetic conditions. A strong geomagnetic disturbance started late on 11 April and all observations described here are obtained during the expansion or recovery phase of the storm. opportunity for that the interval was so disturbed because structure in the plasma complicates the comparison.

Electron temperature values exceed the IRI-95 model results during the entire six hour interval presented in Figure 4. Temperature obtained during night conditions are typically $1000 \mathrm{~K}$ greater than the model results but may be as large as $3000 \mathrm{~K}$ greater than the model at orbital sunrise.

Similar to the 15 February observations, the functional forms of the FPP derived electron density and temperature curves are consistent with the IRI model. Electron density observations are greater than the model results on the undisturbed 15 February interval while often much less than the model predictions on the disturbed 12 April interval.

Observed values of electron temperature exceed the model results in both cases, suggesting either the IRI-95 model under represents electron temperature values (at least for the intervals presented here) or the FPP temperature measurements are high. Input data driving variability in the IRI-95 model is a 13 month smoothed $\mathrm{F} 10.7 \mathrm{~cm}$ radio flux so it is not expected that model results would exactly match observations during any given interval. To determine if the FPP Langmuir probe is accurately providing estimates of the ambient plasma density and temperature values, then, comparison with an average ionosphere model is not the best technique. Independent observations of 


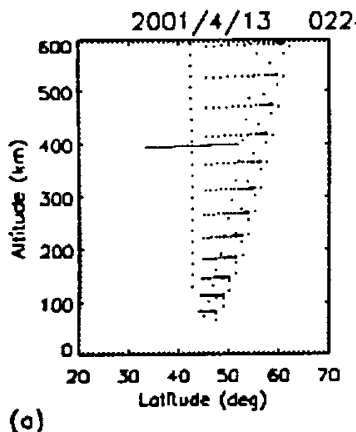

(o)

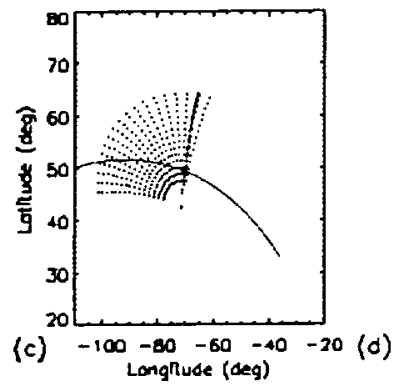

(b)

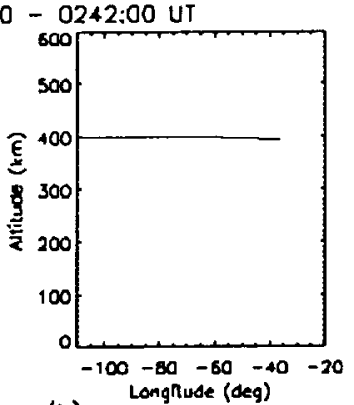

(b)

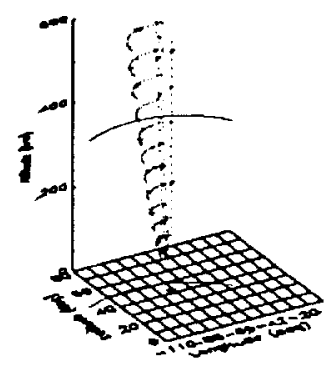

Figure 6. Geometry for an ISS encounter with the radar beam on 13 April 2001 near 02:33 UT including (a) altitude-latitude, (b) altitudelongitude, (c) latitude-longitude, and (d) a 3-D latitude-longitude-altitude plots. Both an azimuth scan and a single zenith profile are present in the 18 minute interval included in the figure. ISS trajectories are plotted at the spacecraft altitude and on the ground plane in the 3-D plot.

electron density and temperature coincident with FPP measurements are required to determine if there is a bias in the FPP measurements.

\section{Comparison to Millstone Hill ISR}

Independent observations of the ionospheric plasma density and temperature were obtained by an incoherent scatter radar (ISR) at the Millstone Hill observatory site (42.1 $1^{\circ} \mathrm{N}$ latitude, $71.9^{\circ} \mathrm{W}$ longitude) from 11 April 2001 through 13 April 2001. Radar operations were conducted in support of a scientific geomagnetic storm study and were fortuitous in providing a number of opportunities for direct comparison of FPP data.

Radar operations included a series of sampling sequences with approximately 52 minutes required to cycle through the complete set of observations. Zenith profiles were obtained once every 20 minutes throughout the storm interval with a series of alternating elevation and azimuth scans providing estimates of plasma parameters as a function of latitude, longitude, and altitude. Information recovered from the radar signals and stored in the site database include the electron and ion densities, electron and ion

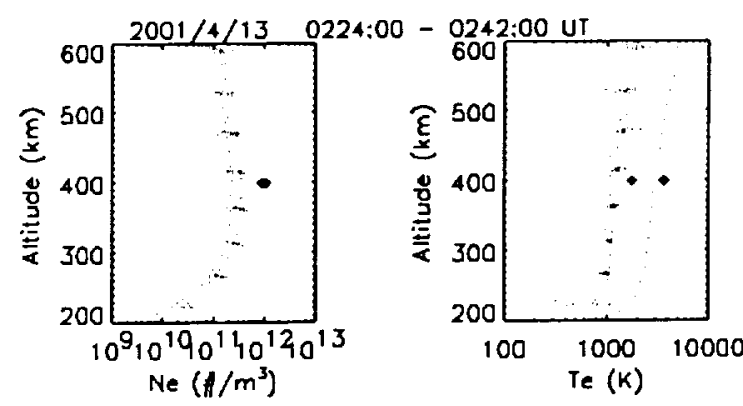

Figure 7. Electron density and temperature profiles for the observations from Figure 6 . Radar values are indicated by the small points and the FPP values are marked with diamonds. The azimuth scan yielded a range of $\mathrm{Ne}$ and $\mathrm{Te}$ values for the different beams while a single profile is present for the zenith profile.

temperatures, plasma convection velocities, neutral temperatures, and a number of other derived parameters. In addition, goodness of fit and errors in measured parameters are also provided in the database although their analysis is not included in the work reported here.

Zenith profiles are obtained by sampling an azimuth of 179 degrees and elevation of 88 degrees above the horizon. Zenith profiles provide the cleanest signature of the altitude variations in plasma parameters because there are no latitude or longitude variations in the data. However, the probability of a spacecraft encountering a zenith profile is very small.

Azimuth scans are obtained by sampling a fixed elevation angle 6 degrees above the horizon over a range of azimuths. For all the observations reported here the radar sampled southwest through west to north of the site at a range of azimuths from -135 degrees to +15 degrees.

An example of the azimuth scan geometry is given in Figure 6 where a part of an azimuth scan is plotted in a selection of formations including altitude-latitude, altitude-longitude, latitude-longitude, and finally a three-dimensional latitude-longitude-altitude plot. Locations of radar range gates are indicated by points in each figure with the ISS trajectory overplotted as a present in Figure 6. Note that the while the spacecraft missed the zenith profile, passing north of the vertical beam, the orbital track penetrated the azimuth beam north and west of the radar site. Direct overpasses of the site are not required due to the extensive range of latitude and longitudes sampled by the radar in the wide area coverage mode used for the geomagnetic storm 


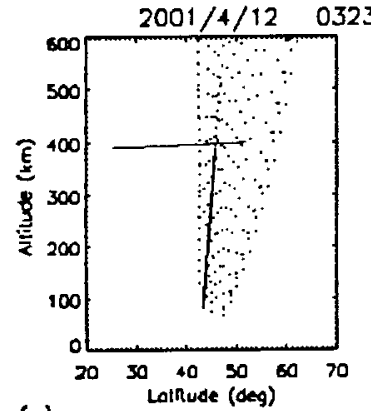

(o)

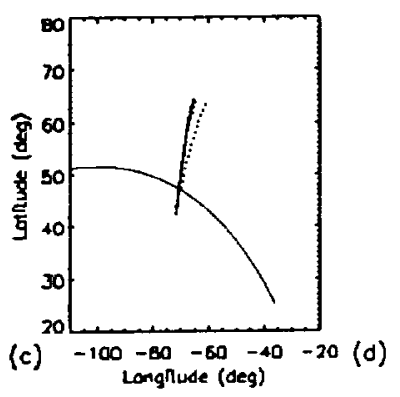

Figure 8. ISS encounter with ISR elevation scan on 12 April 2001 near 03:32 UT. ISS intersects the elevations scan as it passes north of the radar site.

observations. The radar can sample at ISS altitudes beyond $51.6^{\circ} \mathrm{N}$, assuring that any ISS orbit that lies north of the radar will intersect elevation scans. The Extra Wide Coverage mode used in the geomagnetic storm observations during this period provide an excellent probability of ISS encountering the radar beam.

Elevations scans sampled a plane 7 degrees east of the geographic meridian north of the station and 7 degrees west of the geographic meridian south of the station. Minimum elevation angles sampled are approximately 4 degrees above the horizon in the north and 25 degrees above the horizon in the south. At mean ISS altitudes of $400 \mathrm{~km}$ this provides access to latitudes of $58^{\circ} \mathrm{N}$ north of Millstone Hill, exceeding the maximum ISS latitude of $51.6^{\circ} \mathrm{N}$. Latitudes to $36^{\circ} \mathrm{N}$ can be sampled south of the radar site at ISS altitudes.

An example of the ISS encountering an elevation scan beam north of the radar site is shown in Figure 8 and the corresponding $\mathrm{Ne}$ and $\mathrm{Te}$ altitude profiles are given in Figure 9. In this case the radar beam provides a large plane in altitude and latitude through which the vehicle must pass since the radar can sample to higher latitudes than the inclination of the spacecraft.

Finally, an overpass geometry is given in Figure 10 and the corresponding temperature and density altitude

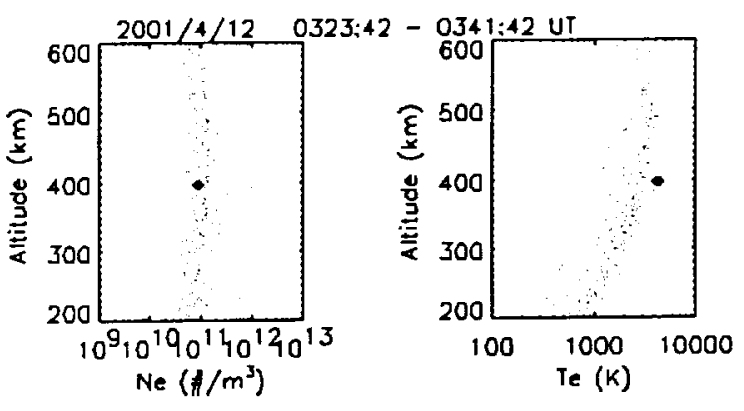

Figure 9. $\mathrm{Ne}, \mathrm{Te}$ altitude profiles from the elevation scan. Density values are similar for both instruments while the FPP temperature exceeds the radar values.

profiles in Figure 11 where the presence of plasma populations with different characteristics is indicated by the differences in temperature and density in the radar beam. The radar elevation scan data for this interval (not shown) indicates the presence of a plasma trough with reduced plasma density and increase temperature poleward of Millstone Hill. These conditions are consistent with plasma characteristics in plasma troughs reported by Foster et al. (1994) where elevated electron temperatures of $-3500 \mathrm{~K}$ were observed in regions where the electron density was reduced by an order of magnitude.

To facilitate comparison of the ISR measurements obtained during overpasses, analysis software was developed to extract average and standard deviations of both FPP and radar derived plasma parameters from near ISS altitudes during close approach to the radar beam. A filter technique employed in the software retained all radar values within a window of 18 minutes centered on the close approach time of the spacecraft to the radar beam. This long interval was required due to the long periods required to complete a single elevation or azimuth scan.

Results from a series of trade studies conducted to determine the impact of retaining FPP data values within windows varying from approximately 1.2 minutes to 10 minutes centered on the close approach time are given in Table 1 . In addition, filters on the Langmuir probe $\chi^{2}$ curve fit values were also employed to eliminate FPP Ne and Te values with poor statistical significance. Minus signs in the table indicate either that no FPP values were available that satisfied the $\chi^{2}$ threshold filter within the required time window or, in the case of the standard deviations, only a single value was available. 

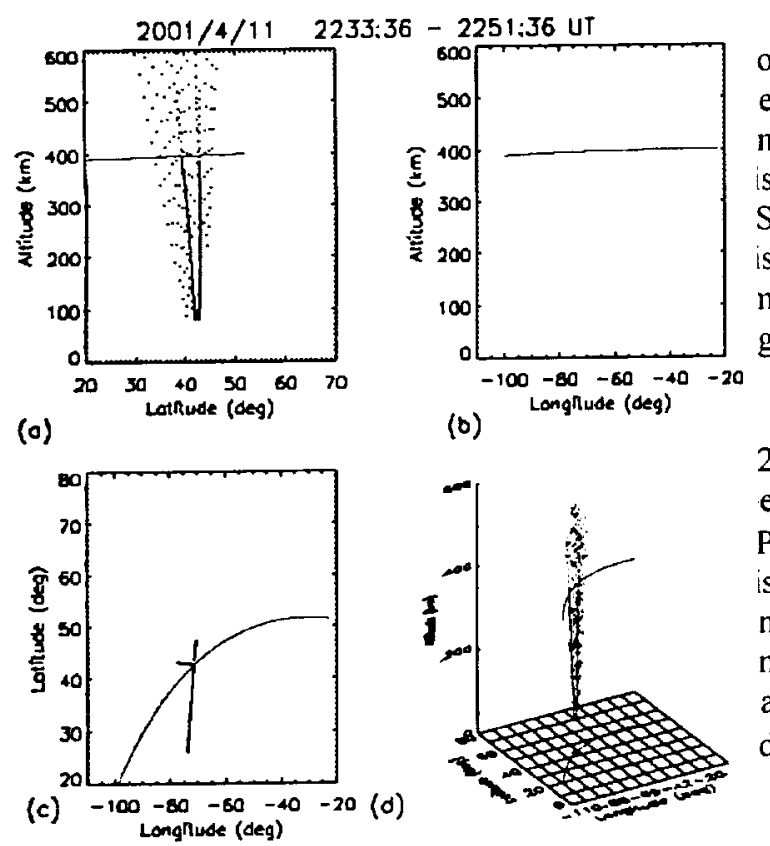

(b)

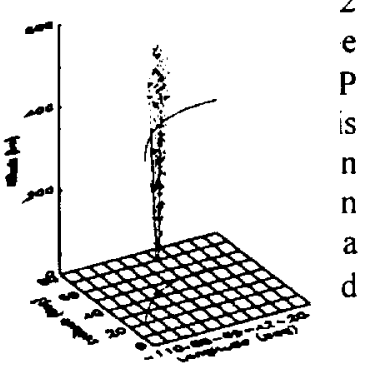

Figure 10. ISS overpass on 11 April 2001 near 22:42 UT. Beams sampling in different directions show large variations in number density and temperatures.

In nearly all cases the FPP temperatures exceed the radar values, often by $1000 \mathrm{~K}$ to $2000 \mathrm{~K}$ or more. The consistency of this difference is striking and is consistent with previous results obtained by Morton et al. (1995) when the FPP instrument (SAMPIE) was flown on the Space Shuttle.

Discrepancies between the FPP and radar observations could have a number of possible origins:

1) Ion current neglected in analysis so additional current term is not properly accounted for in fit (Morton et al., 1995).

2) Errors in the fits could result in excess temperatures. However, the Langmuir Probe $\chi^{2}$ values provide a quantitative measurement of the goodness of fit. Comparing only significant recoveries of $\mathrm{Ne}, \mathrm{Te}$ from the probe curves should avoid any errors due to poor statistics.

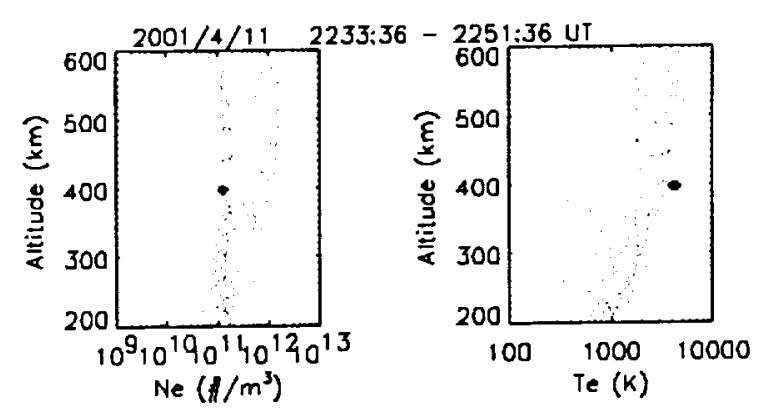

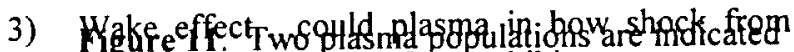

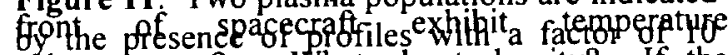

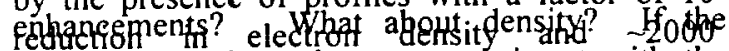

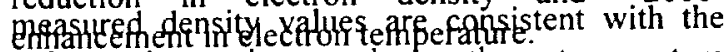
radar observations, then the temperature enhancements are likely not due to wake effects. Need to research this.

4) Drifting electron population affects fits (but $\mathrm{Vd} / \mathrm{Vth}>0.1$ for this to be true....calculate it).

5) Radar values....are they good. Statistical information along with error estimates are available in the radar database but have not been examined to date. In a future analysis we plan to consider in further detail the impact of errors in both the Langmuir probe fits and the radar data results.

\section{Implications for ISS}

The observations reported here suggest a number of implications for future use of plasma instrumentation onboard ISS for engineering support.

Need for coordinated campaigns:

The problems encountered in obtaining coincident observations from the ground based radar and on-orbit instrumentation emphasize the need for a coordinated campaign should DTO's be planned in the future to calibrate ISS instrumentation against ground based data. While the ongoing geomagnetic storm was fortuitous in that Millstone Hill was running a set of wide latitude storm observations, the storm also created conditions that complicated comparison with the FPP and radar $\mathrm{Ne}, \mathrm{Te}$. 
The high latitude of Millstone Hill relative to the ISS inclination assures that elevation scans from Millstone will sample all latitudes up to the maximum ISS latitude. All ISS passes north of Millstone and passes within 36 degrees latitude south of Millstone are available to the radar. Coordination of both the NSF funded radar site as well as the NASA/Russian Space Agency operated ISS facility is required to assure the coincident observations. Scheduling the overpasses of Millstone to assure times over site are either in night or daytime hours would also minimize the possibility of dealing with varying plasma conditions near sunrise and sunset. Plans are underway to request radar time to support such a set of observations when the FPMU is operational onboard ISS, but they will only be valuable if the FPMU is operational during each and every pass through the Millstone beam.

Need for better time resolution data:

The 20 second time resolution of the FPP was minimal

Table 1. Statistical Comparisons of FPP and ISR Results

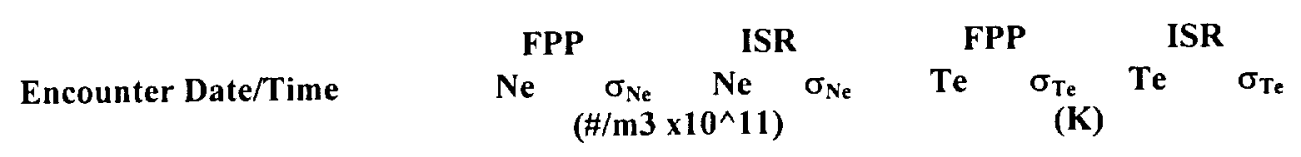

\begin{tabular}{lllrrrrrrrr} 
dT(ISR): & $\mathbf{1 8} \mathbf{~ m i n}$ & dT(FPP): & $\mathbf{1 . 2} \mathbf{m i n}$ & \multicolumn{7}{c}{ Chi-sq $\leq \mathbf{0 . 0 1}$} \\
2001/4/11 & $2233: 36-2251: 36$ UT & 1.26 & 0.05 & 3.32 & 3.93 & 4290 & 91 & 1730 & 1067 \\
$2001 / 4 / 12$ & $0006: 36-0024: 36$ UT & -1.00 & -1.00 & 25.88 & 142.21 & -1 & -1 & 2989 & 1884 \\
$2001 / 4 / 12$ & $0143: 48-0201: 48$ UT & 0.87 & -1.00 & 3.08 & 8.10 & 3602 & -1 & 2453 & 1482 \\
$2001 / 4 / 12$ & $0323: 42-0341: 42$ UT & 0.81 & -1.00 & 1.03 & 1.21 & 4306 & -1 & 2202 & 1680 \\
$2001 / 4 / 12$ & $0500: 00-0518: 00$ UT & 0.85 & -1.00 & 0.79 & 0.42 & 3688 & -1 & 2104 & 1523 \\
$2001 / 4 / 13$ & $0043: 48-0101: 48$ UT & 2.76 & 0.08 & 2.91 & 2.04 & 2485 & 122 & 1651 & 794 \\
$2001 / 4 / 13$ & $0224: 00-0242: 00$ UT & -1.00 & -1.00 & 1.72 & 1.05 & -1 & -1 & 1532 & 941 \\
$2001 / 4 / 13$ & $0401: 48-0419: 48$ UT & 2.79 & -1.00 & 1.31 & 0.79 & 2122 & -1 & 2218 & 839
\end{tabular}

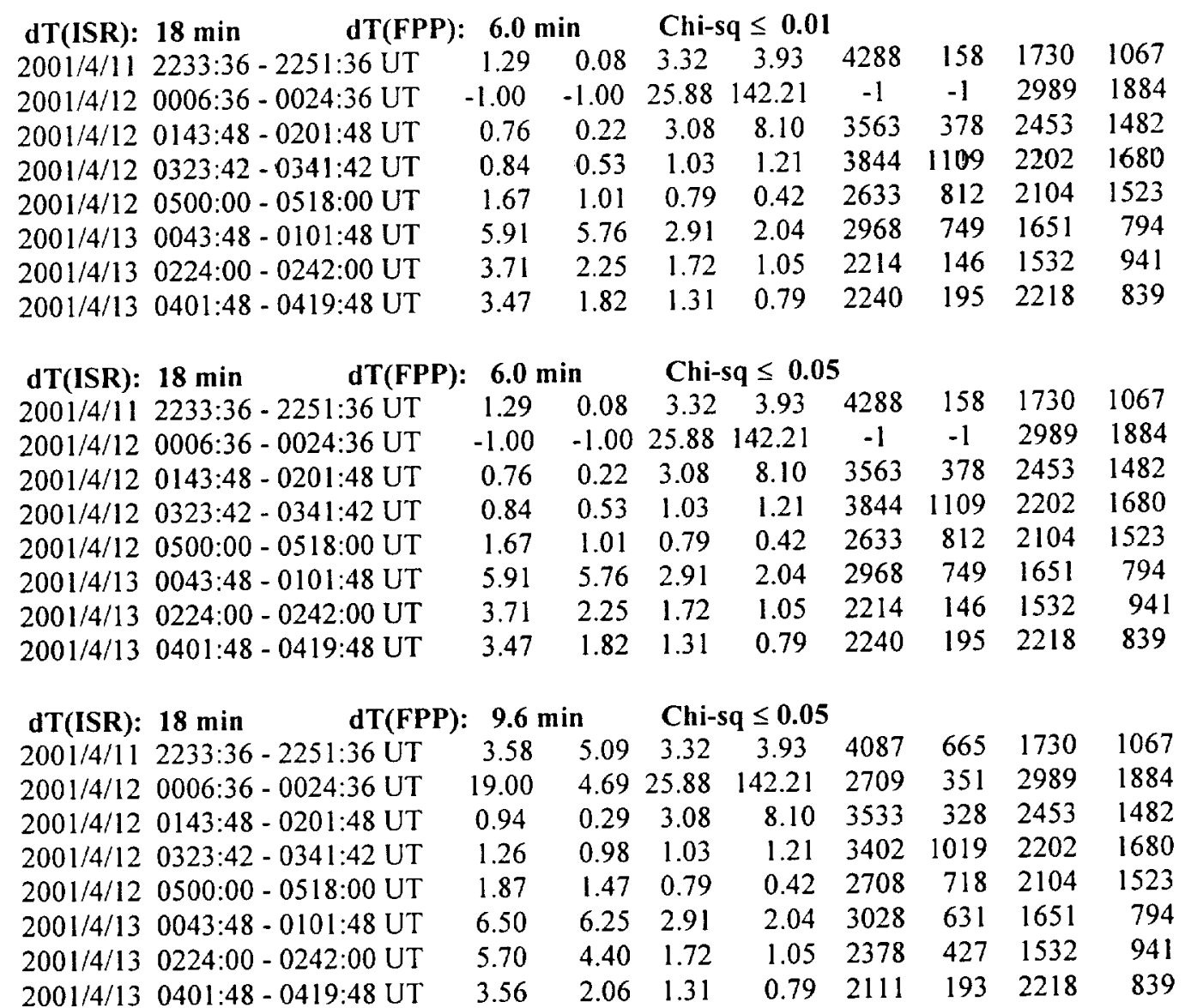


for attempting to compare with the ground site. ISS velocities of $\sim 7 \mathrm{~km} / \mathrm{s}$ puts $140 \mathrm{~km}$ between each data point. In addition, spacecraft charging occurs on millisecond time scales, monitoring for potential enhancements requires much better time resolution than 20 seconds. Finally, should the potential for science from the Floating Potential Monitoring Unit (FPMU), a follow-on instrument to the FPP to be flown in 2003, ever be realized, data rates sufficient to identify structures in plasma density and temperature will be required. Although primarily an engineering support instrument, the added cost and complexity of reporting high time resolution data certainly fits within the Station goals of providing world class science from low Earth orbit.

\section{Need for automated operation:}

Astronaut time was required to operate the data logging for the FPP, scheduling time for both turning the instrument on and off. A number of additional comparison periods would have been present during the 1 January through 1 May period had the instrument been operational during the entire interval. An autonomous operation creates additional opportunities to compare observations with radar experiments as well as ongoing ionosonde operations.

\section{Geomagnetic conditions:}

Comparisons reported here were complicated by the disturbed geomagnetic conditions during the April 1114 period. Future comparisons for calibration purposes would be simplified if attention were made to obtaining the data during quiet intervals. Under these conditions there would be a minimal chance of plasma troughs forming near Millstone Hill and plasma would be relatively unstructured over the range of latitudes sampled by the radar.

\section{Estimating ISS Floating Potential Using Empirical Charging Equations}

Use of the empirical Ferguson-Morton equation to estimate the ISS frame potential (Ferguson, 2001)

$$
\phi=-2.6907 \mathrm{~N}_{\mathrm{e}}{ }^{0.1057} \mathrm{e}^{-8.02 \mathrm{~T} e}
$$

where $\mathrm{Ne}, \mathrm{Te}$, and $\phi$ are the electron density ( $\# / \mathrm{m} 3)$, electron temperature (in $\mathrm{eV}$ ), and $\phi$ the spacecraft potential measured by the FPP. Use of this equation, or similar empirical models as the ISS configuration evolves, has been suggested as a possible technique for predicting the range spacecraft potentials that may be encountered if historical data is used for $\mathrm{Ne}$ and $\mathrm{Te}$ rather than on orbit observations. However, if the FPP temperature observations are consistently high, then the potentials resulting from use of the equation will only be valid if the FPP values are used. Use of ground based radar data, retarding potential analyzer, or other on-orbit plasma density and temperature measurements will predict an error in the potential of magnitude

$$
\begin{aligned}
\phi & =-2.6907 \mathrm{~N}_{\mathrm{e}}{ }^{0.1057} \mathrm{e}^{-8.02(\mathrm{Te}+\Delta \mathrm{Te})} \\
& =\phi_{0} \mathrm{e}^{-8.02 \Delta \mathrm{Te}}
\end{aligned}
$$

where $\phi_{0}$ is the spacecraft potential based correct temperature values. For example, should real electron temperature values approximately $1000 \mathrm{~K}(0.086 \mathrm{eV})$ less than those used to derive the fit be used to predict ISS potentials, the predicted values will be

$$
\phi=\phi_{0} \mathrm{e}^{-8.02(-0.086)}=2 \phi_{0}
$$

half the correct value. It is likely then that use of the Ferguson-Morton relationship with overestimates of the electron temperature is underestimating the range of possible spacecraft potentials.

\section{Conclusions}

Periods where coincident FPP and Millstone Hill observations of the ionospheric plasma have been identified providing an opportunity to compare FPP electron density and temperature measurements with an independent measurement of the same quantities. Comparison of the density values with the IRI-95 model and independent radar measurements obtained during ISS overpass of the Millstone Hill site suggest the FPP density values may be consistent with the model and the radar observatons but the temperature values are consistently high by $1000 \mathrm{~K}$ to $2000 \mathrm{~K}$. Use of FPP values in the empirical Ferguson-Morton equation developed for estimating ISS charging may underestimate the charging hazard on ISS. However, further analysis is necessary to completely understand the difference in the FPP and radar observations, including a careful error analysis of both data sets.

Future work in calibrating Langmuir probes (or any plasma instrument) measurements of ISS plasma environments would benefit from a concerted effort to coordinate with independent measurements like ISR. Difficulties were encountered in obtaining optimal comparions of the two data sets due to the moderate time resolution of the FPP observations and the often inappropriate spacecraft trajectory-radar beam geometry during spacecraft overpasses of the ground site. A dedicated observation program where the radar site optimized radar coverage during spacecraft overpasses would greatly increase the value of the calibration data. 


\section{ACKNOWLEDGEMENTS}

The authors wish to thank Millstone Hill for providing the incoherent radar data. Dst, $\mathrm{Kp}$, and hemispheric power indices are provided by the Space Environment Center, NOAA, U.S. Dept. of Commerce. Funding for this work was provided by a NASA grant for Morton/OAI (NCC3-801) and a NASA contract for Minow/SvT (NAS8-00187).

\section{REFERENCES}

Bilitza, D., K. Rawer, and E. Gruen, Ionospheric Models, Advances in Space Research, Volume 14, Number 12, 1994.

Bilitza, D., International Reference Ionosphere - Status 1995/96, Adv. Space Res. 20, \#9, 1751-1754, 1997.

Carruth, Jr., M.R., T. Schneider, M. McCollum, M. Finckenor, R. Suggs, D. Ferguson, I. Katz, R. Mikatarian, J. Alred, and C. Pankop, ISS and space environment interactions without operating plasma contactor, AIAA Paper \#2001-0401, 2001.

Chaky, R. C., and James C. Lambert, "The ISS plasma contactor," AIAA Paper 96-0627, AIAA, Aerospace Sciences Meeting and Exhibit, 34th, Reno, NV, Jan. 15-18, 1996.

Ferguson, D.C., "Charging of the International Space Station due to its High Voltage Solar Arrays", presented at the $17^{\text {th }}$ Space Photovoltaic Research and Technology Conference, Ohio Aerospace Institute, Cleveland, OH, September, 2001.

Ferguson, D. C., T. L. Morton, and G. B. Hillard, 2001, First Results from the Floating Potential Probe (FPP) on the International Space Station, AIAA-2001-0402.

Ferguson, D. C., D. B. Snyder, and R. Carruth, 1990, Report of the Joint Workshop of the Space Station Freedom Plasma Interactions and Effects Working Group, the Space Station Freedom Plasma Working Group, and the Space Station Freedom EMI/EMC and Electromagnetic Effects Working Group on Evaluation of Impacts of Space Station Freedom Grounding Configurations, May 22-24, 1990. Final Report, Aug. 21, 1990.

Ferguson, D.C., and B. Gardner, Modeling the International Space Station (ISS) floating potentials, AIAA Paper \#2002-0933, 2002.

Foster, J.C., M.J. Buonsanto, M. Mendillo, D. Nottingham, F.J. Rich, and W. Denig, Coordinated stable auroral red arc observations: Relationships to plasma convection, J. Geophys. Res., 99, 11429 11439, 1994.

Medicus, G, "Theory of Electron Collection of Spherical Probes", J. of App. Phys., 32, 2512-2520, 1961.
Medicus, G., "Spherical Langmuir Probe in 'Drifting' and 'Accelerated' Maxwellian Plasmas", J. of App. Phys., 33, 3094 - 3100, 1962.

Morton, T.L., D.C. Ferguson, and G.B. Hillard, "Ionospheric plasma Densities and Temperatures Measured by SAMPIE," AIAA Paper \#1995-0841, 1995.

Perez de la Cruz, C., D. E. Hastings, D. Ferguson, and B. Hillard, "Data analysis and model comparison for the SAMPIE experiment," AIAA Paper \#96-0369, 1996. 